\title{
Comparative analysis of different approaches for dealing with candidate regions in the context of a genome-wide association study
}

\author{
Francesca Lantieri ${ }^{\dagger 1,2}$, Min A Jhun ${ }^{\dagger 3}$, Jungsun Park ${ }^{3}$, Taesung Park*3,4 \\ and Marcella Devoto*1,5,6
}

\begin{abstract}
Addresses: ${ }^{1}$ The Children's Hospital of Philadelphia, 3615 Civic Center Boulevard, Philadelphia, Pennsylvania 19104, USA, ${ }^{2}$ Dipartimento di Scienze della Salute, sezione di Biostatistica, Universita' degli Studi di Genova, via Pastore 1, 16132 Genoa, Italy, ${ }^{3}$ Interdisciplinary Program in Bioinformatics, Seoul National University, Seoul, 56-1 Shillim-Dong, Kwanak-Gu, Seoul, 151-742, Korea, ${ }^{4}$ Department of Statistics, Seoul National University, Seoul, 56-1 Shillim-Dong, Kwanak-Gu, 151-747, Korea, ${ }^{5}$ Department of Pediatrics and CCEB, University of Pennsylvania, 3615 Civic Center Boulevard, Philadelphia, Pennsylvania 19104, USA and ${ }^{6}$ Dipartimento di Medicina Sperimentale, Universita' La Sapienza, Viale Regina Elena 324, 00161, Roma, Italy

E-mail: Francesca Lantieri - f.lantieri@unige.it; Min A Jhun - mhj617@snu.ac.kr; Jungsun Park - pj518@snu.ac.kr;

Taesung Park* - tspark@stats.snu.ac.kr; Marcella Devoto* - devoto@chop.edu

*Corresponding author †Equal contributors
\end{abstract}

from Genetic Analysis Workshop 16

St Louis, MO, USA 17-20 September 2009

Published: 15 December 2009

BMC Proceedings 2009, 3(Suppl 7):S93 doi: 10.1186/1753-6561-3-S7-S93

This article is available from: http://www.biomedcentral.com//753-656I/3/S7/S93

(C) 2009 Lantieri et al; licensee BioMed Central Ltd.

This is an open access article distributed under the terms of the Creative Commons Attribution License (http://creativecommons.org/licenses/by/2.0), which permits unrestricted use, distribution, and reproduction in any medium, provided the original work is properly cited.

\begin{abstract}
Genome-wide association studies (GWAS) test hundreds of thousands of single-nucleotide polymorphisms (SNPs) for association to a trait, treating each marker equally and ignoring prior evidence of association to specific regions. Typically, promising regions are selected for further investigation based on $p$-values obtained from simple tests of association. However, loci that exert only a weak, low-penetrant role on the trait, producing modest evidence of association, are not detectable in the context of a GWAS. Implementing prior knowledge of association in GWAS could increase power, help distinguish between false and true positives, and identify better sets of SNPs for follow-up studies.

Here we performed a GWAS on rheumatoid arthritis (RA) patients and controls (Problem I, Genetic Analysis Workshop 16). In order to include prior information in the analysis, we applied four methods that distinctively deal with markers in candidate genes in the context of GWAS. SNPs were divided into a random and a candidate subset, then we applied empirical correction by permutation, false-discovery rate, false-positive report probability, and posterior odds of association using different prior probabilities. We repeated the same analyses on two different sets of candidate markers defined on the basis of previously reported association to RA following two different approaches. The four methods showed similar relative behavior when applied to the two sets, with the proportion of candidate SNPs ranked among the top 2,000 varying from 0 to $100 \%$. The use of different prior probabilities changed the stringency of the methods, but not their relative performance.
\end{abstract}




\section{Background}

Genome-wide association studies (GWAS) to identify the genetic risk factors underlying complex disease are now feasible thanks to advances in genotyping technology and the development of commercial products featuring panels with hundreds of thousands of singlenucleotide polymorphisms (SNPs). A common approach is to use the GWAS design to detect "promising" traitassociated regions that could undergo further investigation. Typically, the top ranked markers are selected for follow-up analysis based on p-values obtained from simple tests of association, such as the 1 degree-offreedom chi-square test on allele frequency difference between cases and controls. For several diseases, some candidate genes may have already been identified by linkage or association studies, or can be suggested on the basis of functional or other biological, rather than statistical, evidence. Such loci may exert only a weak, low-penetrance role on the trait, producing modest evidence of association. In the context of a genomewide study, the significance of markers in these regions could be low and thus undetectable. GWAS typically ignore any prior knowledge that may support evidence of association to specific regions by treating each marker equally. Incorporating this information into GWAS could increase power, help distinguish between false and true positives, and identify better informed sets of SNPs for follow-up studies.

The HLA-DRB1 gene has long been known to be a major rheumatoid arthritis (RA) susceptibility locus [1]. More recently, variants of the PTPN22 gene have been reported to be associated to RA $[2,3]$. Common genetic variants at TRAF1 and C5 [4] and a haplotype at STAT4 have been described in association to RA [5]. Besides these, several other genes have been proposed and tested for association to RA with controversial results.

Different ways to deal with markers in candidate genes in the context of genome-wide studies have been suggested [6-8]. Here we selected specific genomic regions as RA candidate loci using two different approaches and compared the relative performance of some of these methods applied to the RA dataset analyzed by Plenge et al. [4] (Problem 1 of Genetic Analysis Workshop 16 (GAW16)).

\section{Methods \\ Candidate regions}

On the basis of previously reported association to RA and following two different approaches, we have defined two sets of RA candidate markers: a more inclusive and exhaustive "gene-based" set, and a more selective "SNPbased" set.
Candidate Subset 1 included 64 genes defined as associated to the broad phenotype of RA in the Genetic Association Database $[9,10]$. SNPs included in the prioritized subset were those located in a candidate gene and a region of $5 \mathrm{~kb}$ on either side of it.

Set 2 was chosen through a review of recently (20062008) published studies of RA [2-5]. Markers described in these papers or in their references were included in the Set 2. There were more than 50 SNPs reported as showing association with RA, among which 24 SNPs were genotyped in GAW16. These SNPs and 14 additional ones with pair-wise $r^{2}$ with them greater than 0.5 were selected, leading to a total of 38 candidate SNPs. In addition, we searched RA-related SNPs from NCBI OMIM database [11] and retrieved from the HapMap data those SNPs whose pair-wise $r^{2}$ with them greater than 0.5 , for a total of 51 genes. Thus, 89 SNPs were included in the prioritized subset, and the remaining SNPs were defined as the non-prioritized subset.

We excluded from all analyses the HLA region genes on chromosome 6 (from 29,794,096 bp to 33,209,868 bp) because HLA genes, and in particular DR alleles, have already been unequivocally demonstrated to be strongly associated to RA.

The genotypes at 545,080 SNPs from the Illumina 550k chip were available for 868 North American Rheumatoid Arthritis Consortium cases and 1194 controls. SNPs that failed genotyping in more than $10 \%$ of all individuals or presenting a minor allele frequency (MAF) lower than $1 \%$ were discarded. Missing genotypes at more than $10 \%$ of markers was set as a sample exclusion condition, but none of the individuals were removed.

After filtering for low MAF and low genotyping rate, and excluding markers from the HLA region, 515,179 SNPs remained. Of these, 681 SNPs localized in 55 candidate genes in Set 1, and 72 were among the candidate SNPs in Set 2. The complete lists of SNPs and genes included in the two prioritized subsets are available from the authors. The complementary subsets of markers were considered to be random SNPs routinely genotyped in GWAS.

\section{Statistical methods}

We have applied four different methods to prioritize results from candidate regions in the context of a genome-wide study, in addition to standard GWAS.

These methods included a Bayesian approach to calculate posterior odds for association (henceforth referred to as PO), assigning different prior probabilities to the 
selected candidate marker subset and to the routinely genotyped marker subset [6]. Initially, we specified a prior probability of $p=0.1$ for the candidate region markers and of $p=0.00018$ for the other markers (PO1). These values were chosen so that a candidate marker significant at $p=0.01$ and a routine marker significant at $p=0.00001$ yield similar values for the PO, as suggested by Curtis et al. [6]. To investigate the effect of different prior probabilities, we also assigned a less confident prior probability of association of $p=0.01$ to the candidate SNPs subset (PO2).

Next we used the approach based on the false-positive report probability (FPRP) described by Wacholder et al. [7]. The FPRP is the probability of having no true association between a genetic variant and disease given a statistically significant finding. This can be calculated based on the prior probability of real association, the observed $p$-value, and the statistical power of the study, which in turn depends on the sample size and the oddsratio under the alternative hypothesis. The odds-ratio under the alternative hypothesis was fixed at 1.2 and two different prior probabilities of 0.1 (FPRP1) and 0.01 (FPRP2) for candidate gene markers and 0.00018 for random markers were considered. We used the formula described by Wacholder et al. [7] to calculate the FPRP.

We also utilized a prioritized subset analysis (PSA) and applied the false-discovery rate (FDR) procedure separately to the subset of candidate markers and to the complementary subset of random markers, as suggested by Li et al. [8].

Finally, we calculated empirically corrected $p$-values by permutation for the whole subset of random SNPs and for the candidate SNP subset separately (henceforth referred to as EMP), by swapping the case/control label of individuals (assuming they are interchangeable under the null). A total of 10,000 permutations were ran on each SNP by means of the $(\max ) \mathrm{T}$ procedure implemented in the software PLINK $[12,13]$. This procedure controls for the number of SNPs tested by comparing each observed test statistic against the maximum of all permuted statistics (i.e., over all SNPs) for each single replicate.

Standard case-control genome-wide association analysis was carried out using the 1-df allelic test from PLINK [13]. To implement the PO1 and PO2 procedures, $p$-values were converted into $\mathrm{PO}$ values for association using software provided by Curtis et al. [6,14]. The FPRP was calculated using software provided by Wacholder et al. [7]. To apply the FDR recommended by Li et al. [8], the procedure described by Benjamini and Hochberg [15] and implemented in PLINK was used.

\section{Results}

The genome-wide analysis conducted on the 515,179 SNPs that passed quality control revealed that 66 SNPs were associated to RA with allelic test $p$-values $<10^{-7}$, which remained significant at $\alpha=0.05$ after Bonferroni correction for multiple tests. None of those SNPs was located in any of the 55 selected candidate genes of Set 1 , while six were included in the candidate Set 2 (rs10760130, rs1953126, rs2900180, rs3761847, and rs881375 in the region around TRAF1 on chromosome 9, and rs2476601 in PTPN22 on chromosome 1).

To identify regions worth of further analyses in followup studies, we arbitrarily chose the first 2,000 SNPs based on the ranking derived by the standard analysis and the other approaches described in the Methods section. Results thus obtained within each of the two candidate sets are compared.

\section{Candidate set I}

The 2,000 most significant SNPs following standard genome-wide analysis had allelic test $p$-values that ranged between $3.25 \times 10^{-4}$ and $1.10 \times 10^{-15}$. Even though not significant at the genome-wide level, two SNPs from this group were located in candidate genes, specifically rs6435203 in CTL4A $\left(p=2.56 \times 10^{-4}\right)$ and rs10480340 in TRB@ $\left(p=1.70 \times 10^{-4}\right)$.

When applying the FDR procedure separately in the candidate SNP subset and in the complementary subset of random markers [8], none of the candidate SNPs ranked among the top 2,000 or had FDR $<0.05$. The list of the 2,000 top-ranked SNPs obtained by PSA/FDR was almost identical to that obtained on the basis of their nominal $p$-values. Following separate permutation analysis on the subset of random SNPs and on the candidate SNP subset (EMP), only 575 SNPs showed an empirical $p$-value lower than 1 and could thus be ranked, including 39 SNPs from 22 candidate genes. In the ranking based on the PO values, specifying a prior probability of $p=0.1$ for candidate gene markers and of $p=0.00018$ for the other routinely genotyped markers [6], 348 of the 2,000 top ranked SNPs were located in 49 of the candidate genes. Using a prior probability of $p=0.01$ for the candidate gene markers reduced their number to 40 SNPs from 19 genes. All 681 candidate gene SNPs (with just one with FPRP less than 0.05) were included in the top 2,000 markers using FPRP1, and only 152 using FPRP2 [7] (Figure 1).

In conclusion, 3,318 different SNPs were ranked among the first 2,000 SNPs by at least one method, including all 681 SNPs located on the selected candidate genes. Only 345 SNPs were ranked among the top 2,000 by all methods, and none of these were located on a candidate gene. 

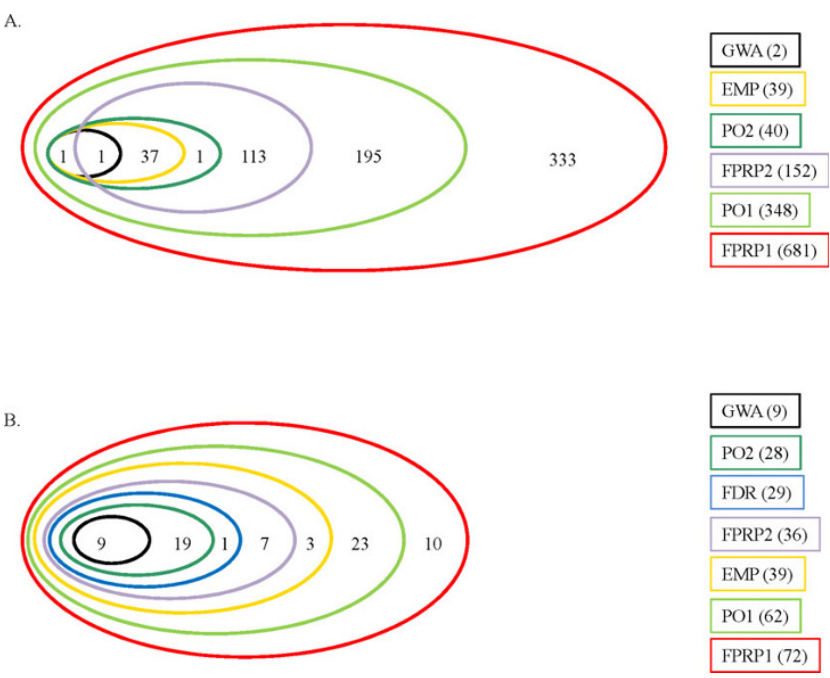

Figure I

Top ranked SNPs detected by each method. Venn diagram with the number of candidate SNPs ranked among the top 2,000 markers by each method in Candidate Sets I (A) and 2 (B). GWA, standard allelic test; FDR, PSA followed by FDR [8]; EMP, PSA followed by empirical correction; PO I and PO2, posterior odds [6] with prior probabilities for candidate SNPs of 0.1 and 0.01 ; FPRPI and FPRP2, falsepositive report probability [7] with prior probabilities for candidate SNPs of 0.1 and 0.01 .

\section{Candidate set 2}

Nine candidate SNPs from Set 2 were among the top 2,000 SNPs ranked with the standard genome-wide analysis.

The PSA/FDR method increased the number of candidate SNPs among the top ranked to 29 , with the inclusion of 20 additional SNPs, with nominal $p$-values ranging from $3 \times 10^{-2}$ to $1 \times 10^{-12}$. Of these, 26 had FDR $<0.05$, while in the standard analysis only eight candidate SNPs had significant FDR.

Ranking based on p-values empirically corrected by permutation for the subsets of non-candidate and candidate SNPs separately raised the number of candidate SNPs among the top ranked SNPs to 39, including 11 with empirical $p$-values lower than 0.05. In this analysis, the total number of SNPs with empirical p-values lower than 1 was 500 .

The number of top-ranked candidate SNPs further increased to 62 based on the POs ranking under PO1. Under PO2, this number was only 28.

Using FPRP1, all 72 candidate SNPs were included among the top 2,000, although only 14 had FPRP $<0.05$. When the prior probability of the candidate SNPs was changed to 0.01 (FPRP2), only 36 of them were retained. In conclusion, the PO method was more stringent than the FPRP method under both sets of prior probabilities (Figure 1).

Overall, 2,798 different SNPs were ranked among the first 2,000 SNPs by at least one method, including all 72 candidate SNPs. Nine candidate SNPs in particular were among the 319 SNPs ranked among the top 2,000 by all methods.

\section{Discussion}

With respect to selection of SNPs for follow-up studies, the standard genome-wide analysis yielded the lowest number of candidate SNPs among the top 2,000 for all the methods applied to Set 2, while the PSA/FDR method was even less inclusive for Set 1 . The application of methods designed to prioritize results from selected regions generally increased the number of candidate SNPs that would undergo follow-up analyses. The four methods showed the same relative performance in the two candidate subsets, with the PSA/FDR approach being the least inclusive, followed by the empirical correction performed separately on the candidate subsets and the random subsets, and finally by the POs method using the prior probability $p=0.1$. In both candidate subsets, the FPRP approach with prior probability set at 0.1 ranked all candidate SNPs among the top 2,000 markers and was thus more permissive than the other methods.

The prior probabilities used for PO1 and FPRP1 (0.1 for candidate SNPs and 0.00018 for random SNPs) result in a 556-fold greater confidence on causality of candidate SNPs compared to random SNPs. To evaluate the impact of these parameters, we also considered a less confident prior for the candidate SNP of $p=0.01$. Under these conditions, PO2 and FPRP2 were less inclusive, as expected, yielding an even smaller number of candidate SNPs among the top 2,000 than EMP. However, their relative performance remained unchanged.

The different methods in Set 2 provided more homogenous results than in Set 1 (Figure 1). The number of candidate SNPs that were ranked among the top 2,000 by the different methods ranged from 0 to 681 (100\%) in Set 1, and from $28(39 \%)$ to $72(100 \%)$ in Set 2. When ranking the top 1,000 SNPs only, those figures ranged from $0 \%$ to $63 \%$ in Set 1 and from $28 \%$ to $97 \%$ in Set 2, indicating that the candidate SNPs from Set 2 were more highly ranked on average than those from Set 1. In addition to comprising a smaller number of SNPs, our hypothesis is that this may be due to the fact that Set 2 included stronger candidates following a more careful review of the recent literature, while Set 1 included every 
gene reported at least once in the literature as associated to RA. Specifically, Set 2 was SNP-based, so that actual SNPs reported as associated with RA were investigated (or SNPs in linkage disequilibrium with them), while Set 1 was gene-based, so that SNPs localized in a candidate gene, but not necessarily related to RA, could have lowered the power to detect association. As a matter of fact, only $10 \%$ of SNPs from the Candidate Set 1 had nominally significant $p$-values $<0.05$, while $42 \%$ of SNPs from Set 2 were nominally significant at the same $\alpha$.

Bayesian methods are particularly intriguing as a way to deal with multiple tests in GWAS. It has been claimed that in interpreting observed associations the prior credibility of the hypotheses is more relevant than the number of tests performed [16]. The methods by Curtis et al. [6] and Wacholder et al. [7], while inspired by a Bayesian approach, are not fully Bayesian. They do not specify a prior distribution for the effect size, which is assumed as known in the FPRP method (dichotomized as presence or absence of effect) [7]. Curtis et al. [6] instead used the ratio of likelihoods maximized under the alternative and null hypotheses as proxies for the distribution that would have been obtained over the universe of alternative and null hypotheses. In this light, additional methods, such as the Bayesian false-discovery probability recently proposed by Wakefield et al. [17] should be considered and investigated as an interesting alternative approach.

\section{Conclusion}

The results obtained here are dependent on a number of arbitrary choices, such as the set of candidate genes and SNPs selected, the prior probabilities used in the analyses, and the number of SNPs selected for followup analyses. Given the present conditions, the relative performance of the methods tested was similar in the two candidate subsets, even though the selection criteria for the two sets were very different. However, in neither of the two candidate subsets do we know which variant is truly disease-associated. Thus we cannot say if the association with candidate markers detected using these methods reflects a real increase in power to detect weak but true association. More detailed analyses of simulated data under different scenarios for variable ranges of the model parameters are needed to better evaluate these methods' relative performances.

\section{List of abbreviations used}

EMP: Empirical $p$-value calculated by permutation; FDR: False-discovery rate; FPRP: False-positive report probability; GAW16: Genetic Analysis Workshop 16; GWAS: Genome-wide association study; MAF: Minor allele frequency; PO: Posterior odds; PSA: Prioritized subset analysis; RA: Rheumatoid arthritis; SNPs: Single-nucleotide polymorphisms

\section{Competing interests}

The authors declare that they have no competing interests.

\section{Authors' contributions}

FL participated in the design of the study, selected the candidate gene Set 1, performed the statistical analysis and drafted the manuscript. MAJ participated in the design of the study, selected the candidate gene Set 2, performed the statistical analysis, and helped to draft the manuscript. JP performed the raw data preprocessing participated in the data analysis and discussion followed. TP conceived of the study, participated in its design and coordination, and helped to draft the manuscript. MD conceived of the study, participated in its design and coordination, and helped to draft the manuscript. All authors read and approved the final manuscript.

\section{Acknowledgements}

The Genetic Analysis Workshops are supported by $\mathrm{NIH}$ grant ROI GM03 I575 from the National Institute of General Medical Sciences. TP is supported by the National Research Laboratory Program of Korea Science and Engineering Foundation (MI0500000126). The authors thank Mark Bowser for revising the manuscript.

This article has been published as part of BMC Proceedings Volume 3 Supplement 7, 2009: Genetic Analysis Workshop 16. The full contents of the supplement are available online at http://www.biomedcentral.com/ $|753-656| / 3$ ? issue=S7.

\section{References}

I. Gregersen PK, Silver J and Winchester RJ: The shared epitope hypothesis: an approach to understanding the molecular genetics of susceptibility to rheumatoid arthritis. Arthritis Rheum 1987, 30:1205-1213.

2. Hinks A, Worthington J and Thomson W: The association of PTPN22 with rheumatoid arthritis and juvenile idiopathic arthritis. Rheumatology (Oxford) 2006, 45:365-368.

3. The Wellcome Trust Case Control Consortium: Genome-wide association study of 14,000 cases of seven common diseases and 3,000 shared controls. Nature 2007, 447:66I-678.

4. Plenge RM, Seielstad M, Padyukov L, Lee AT, Remmers EF, Ding B, Liew A, Khalili H, Chandrasekaran A, Davies LR, Li W, Tan AK, Bonnard C, Ong RT, Thalamuthu A, Pettersson S, Liu C, Tian C, Chen WV, Carulli JP, Beckman EM, Altshuler D, Alfredsson L, Criswell LA, Amos Cl, Seldin MF, Kastner DL, Klareskog L and Gregersen PK: TRAFI-C5 as a risk locus for rheumatoid arthritis-a genomewide study. N Engl J Med 2007, 357: $1199-1209$.

5. Remmers EF, Plenge RM, Lee AT, Graham RR, Hom G, Behrens TW, de Bakker PI, Le JM, Lee HS, Batliwalla F, Li W, Masters SL, Booty MG, Carulli JP, Padyukov L, Alfredsson L, Klareskog L, Chen WV, Amos Cl, Criswell LA, Seldin MF, Kastner DL and Gregersen PK: STAT4 and the risk of rheumatoid arthritis and systemic lupus erythematosus. N Engl J Med 2007, 357:977-986.

6. Curtis $D$, Vine $A E$ and Knight J: A pragmatic suggestion for dealing with results for candidate genes obtained from genome wide association studies. BMC Genet 2007, 8:20.

7. Wacholder S, Chanock S, Garcia-Closas M, El Ghormli L and Rothman N: Assessing the probability that a positive report is 
false: an approach for molecular epidemiology studies. J Natl Cancer Inst 2004, 96:434-442.

8. Li C, Li M, Lange EM and Watanabe RM: Prioritized subset analysis: improving power in genome-wide association studies. Hum Hered 2008, 65:|29-|4|.

9. Genetic Association Database. http://geneticassociationdb.nih. govl.

10. Becker KG, Barnes KC, Bright TJ and Wang SA: The genetic association database. Nat Genet 2004, 36:43|-432.

II. OMIM. http://www.ncbi.nlm.nih.gov/omim.

12. PLINK...Whole genome association analysis toolset. http:// pngu.mgh.harvard.edu/ purcell/plink/.

13. Purcell S, Neale B, Todd-Brown K, Purcell Thomas L, Ferreira MA, Bender D, Maller J, Sklar P, de Bakker PI, Daly MJ and Sham PC: PLINK: a toolset for whole-genome association and population-based linkage analysis. Am J Hum Genet 2007, 81:559-575.

14. Posterior Odds Calculation Software. http://www.smd.qmul. ac.uk/statgen/avine/software.html.

15. Benjamini $Y$ and Hochberg $Y$ : Controlling the false discovery rate: a practical and powerful approach to multiple testing. J Royal Stat Soc Ser B 1995, 57:289-300.

16. Thomas DC and Clayton DG: Betting odds and genetic associations. I Natl Cancer Inst 2004, 96:42 I-423.

17. Wakefield J: A Bayesian measure of the probability of false discovery in genetic epidemiology studies. Am J Hum Genet 2007, 81:208-227.
Publish with Biomed Central and every scientist can read your work free of charge

"BioMed Central will be the most significant development for disseminating the results of biomedical research in our lifetime."

Sir Paul Nurse, Cancer Research UK

Your research papers will be:

- available free of charge to the entire biomedical community

- peer reviewed and published immediately upon acceptance

- cited in PubMed and archived on PubMed Central

- yours - you keep the copyright
BioMedcentral 